\title{
A THEORY OF MANIPULATION AND CONTROL FOR MICROFABRICATED ACTUATOR ARRAYS
}

\author{
Karl-Friedrich Böhringer Bruce R. Donald \\ Robotics \& Vision Laboratory* \\ Department of Computer Science \\ Cornell University \\ Ithaca, NY 14853, USA
}

\begin{abstract}
This paper investigates manipulation tasks with arrays of microelectromechanical structures (MEMS). We develop a model for the mechanics of microactuators and a theory of sensorless, parallel manipulation, and we describe efficient algorithms for their evaluation.

The theory of limit surfaces offers a purely geometric characterization of micro-scale contacts between actuator and moving object, which can be used to efficiently predict the motion of the object on an actuator array. We develop a theory of sensorless manipulation with microactuator arrays. It is shown how simple actuator control strategies can be used to uniquely align a part up to symmetry. These manipulation strategies can be computed efficiently and do not require sensor feedback. This theory is applicable to a wide range of microactuator arrays. Our actuators are oscillating structures of single-crystal silicon fabricated in a low-temperature SCREAM process. They exhibit high aspect ratios and high vertical stiffness, which is of great advantage for an effective implementation of our theory. Calculations show that arrays of these actuators can generate forces that are strong enough to levitate and move e.g. a piece of paper.
\end{abstract}

\section{INTRODUCTION}

A wide variety of micromechanical structures (devices typically in the $\mu m$ range) has been built by using processing techniques developed in the VLSI industry. Various microsensors and actuators have been shown to perform successfully. E.g. a singlechip air-bag sensor is commercially available [1]; video projections using an integrated, monolithic mirror array have been demonstrated recently [16]. More difficult is the fabrication of microrobotic devices that can interact and actively change their environment. Problems arise from (1) unknown material properties and the lack of adequate models for mechanisms at very small scales, (2) the limited range of motion and force that can be generated with microactuators, (3) the lack of sufficient sensor information with regard to manipulation tasks, and (4) design limitations and geometric tolerances inherent in the fabrication process. This paper addresses in particular the first three points.

\footnotetext{
${ }^{*}$ This paper describes research done in the Robotics and Vision Laboratory at Cornell University. Support for our robotics research is provided in part by the National Science Foundation under grants No. IRI-8802390, IRI-9000532, IRI-9201699, and by a Presidential Young Investigator award to Bruce Donald, and in part by the Air Force Office of Sponsored Research, the Mathematical Sciences Institute, Intel Corporation, and AT\&T Bell laboratories.

${ }^{\dagger}$ This work was supported by ARPA under contract DABT 63-69-C0019. The device fabrication was performed at the National Nanofabrication Facility (NNF), which is supported by the NSF grant ECS-8619049, Cornell University, and Industrial Affiliates.
}

\author{
Robert Mihailovich Noel C. MacDonald \\ School of Electrical Engineering and \\ The National Nanofabrication Facility ${ }^{\dagger}$ \\ Cornell University \\ Ithaca, NY 14853, USA
}

We are interested in computational tools for the design, analysis, and control of MEMS. Based on work on sensorless and near-sensorless manipulation $[5,7]$, we have developed geometric theories of manipulation and control for microactuator arrays, and we have developed and implemented efficient algorithms for their evaluation. Penecontemporaneously we have designed, built, and tested microfabricated actuators (length, width $50 \mu \mathrm{m}$ to $200 \mu \mathrm{m}$ ) in the National Nanofabrication Facility at Cornell University $[18,19,12]$. Our calculations show that arrays of these actuators are strong enough to accomplish practical manipulation tasks.

The next section briefly introduces microfabricated actuator arrays. In Section 3 we investigate manipulation strategies for microactuator arrays. A model for individual actuators and their interaction with a movable object is described in Section 4 . Section 5 describes the design, fabrication, and analysis of our devices in more detail. Conclusions and an outlook on future work follow in Section 6 .

\section{MICROFABRICATED ACTUATOR ARRAYS}

Several kinds of devices to position small objects in the plane have been presented recently. Pister et al. [15] use an air cushion generated by microfabricated nozzles to levitate objects, and move them with electrostatic forces. Takeshima and $\mathrm{Fu}^{-}$ jita [17] introduce the concept of a distributed micro motion system (DMMS) that consists of an array of cooperating actuator modules. Furuhata et al. [6] have built arrays of ultrasonic microactuators. Konishi and Fujita [11] use air flow controlled by microvalves to both levitate and move objects. Ataka et al. [2] use thermobimorph cantilever beams to mimic the motion and function of cilia. Due to low friction in the air bearing, motion induced with designs [15] and [11] is fast but hard to control because of the lack of damping. Design [2] allows more control but operates at low frequencies $(\approx 1 H z)$. Our design is closest to Furuhata's [6], with slightly larger devices and a larger range of out-of-plane motion. It combines controlled actuator-object interaction with high operation speed. Our actuators are based on microfabricated torsional resonators [12]. A torsional resonator is a rectangular grid etched out of singlecrystal silicon and suspended by two rods that act as torsional springs (Figure 1a). When an AC voltage is applied between grid and adjacent electrodes, the grid oscillates at resonance frequencies in the high $k H z$ range, the edges of the grid reaching amplitudes of several $\mu \mathrm{m}$ (Figure 1b). Our calculations have shown that the forces generated with an array of torsional resonators are large enough to levitate e.g. a piece of paper (see Section 5).

By introducing asymmetries into the resonator grid (such as placing the torsional rods off the center of the grid, or adding 


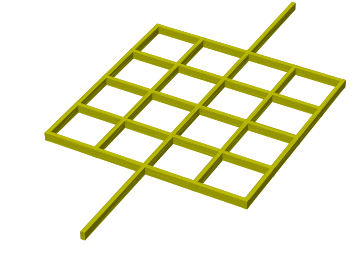

(a)

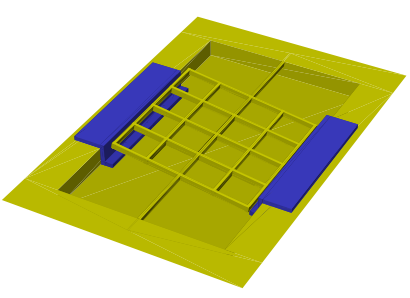

(b)

Figure 1: Torsional resonator (CAD model): (a) Resonator grid with suspending beams. (b) Resonator and electrodes (in dark color).

poles on one side of the grid) anisotropic lateral forces are generated, thus achieving a motion bias for the object on top of the actuator.

Each actuator can generate motion in one specific direction if it is activated; otherwise it acts as a passive frictional contact. The combination and selective activation of several actuators with different motion bias allows us to generate various motions in the plane. Figure 2 shows such a "motion pixel."

Design aspects, fabrication process and mechanism analysis are described in more detail in section 5 .

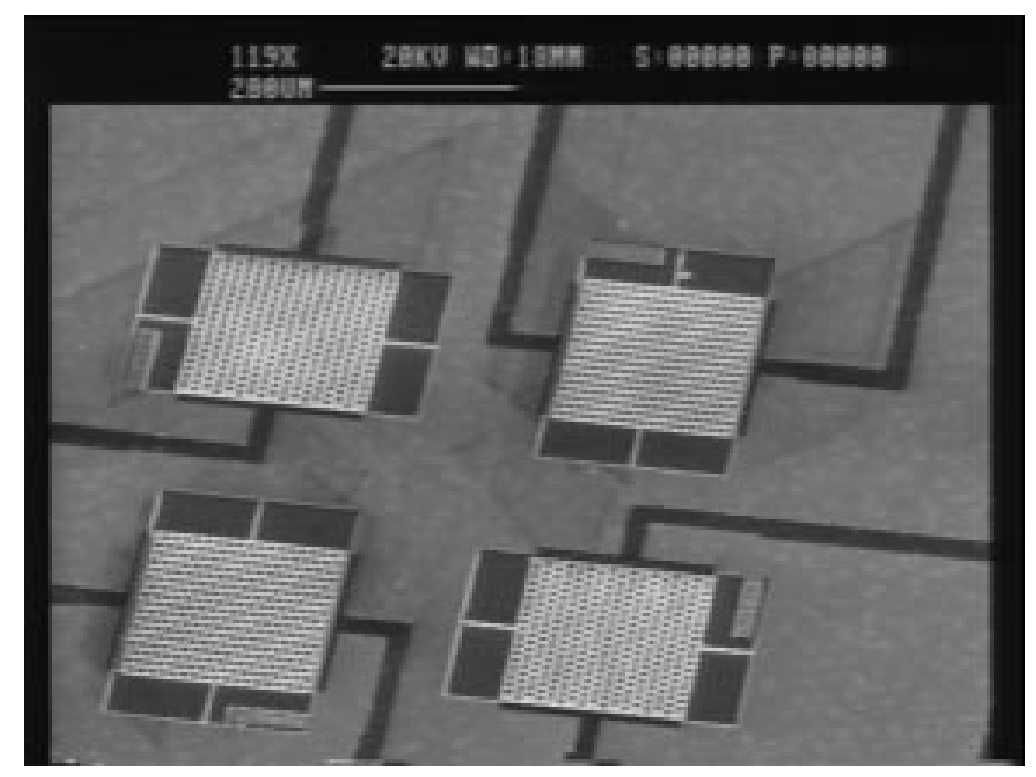

Figure 2: Prototype motion pixel (SEM micrograph).

\section{MACROSCOPIC MODEL FOR MANIPULATION}

In this section we develop a geometric theory of manipulation for microactuator arrays. Our ideas are based on the groundbreaking work of Erdmann and Mason [5] in the field of sensorless and near-sensorless manipulation. In this line of work, Peshkin and Sanderson [14] have shown how to align parts on a conveyer belt with stationary fences. Goldberg [7] has given an algorithm to align parts by a sequence of grasps with a parallel-jaw gripper. In the following we show how, under reasonable assumptions, the problem of aligning a part with a microactuator array can be reduced to the alignment task with a parallel-jaw gripper, effectively using the actuator array to simulate a two-finger gripper.

Goldberg's algorithm [7] takes the geometry of an arbitrary polygonal part $P$ and determines its squeeze function $s: \mathbb{S}^{1} \rightarrow$ $\mathbb{S}^{1}$, where $\mathbb{S}^{1}$ is the set of planar orientations. ${ }^{1}$ The squeeze

\footnotetext{
${ }^{1} \mathbb{S}^{1}$ can be viewed as the circular interval $[0,2 \pi)$, where all expressions are evaluated modulo $2 \pi$.
}

function describes the change in orientation of $P$ when it is grasped by a parallel-jaw gripper with negligible friction. It assumes that the jaws make contact with the part simultaneously, and that the part rotates until the distance between the jaws reaches a local minimum (squeeze grasp). The squeeze function can be derived from the diameter function $d: \mathbb{S}^{1} \rightarrow \mathbb{R}$, which describes the distance between the two horizontal lines tangential to $P$ at a particular orientation. The squeeze function maps all orientations that lie between two adjacent 1ocal maxima of the diameter function to the orientation corresponding to the intermediate local minimum (Figures $3 \mathrm{a}, \mathrm{b}$, c). Goldberg then gives an algorithm that, given a specific squeeze function, computes a sequence of grasp orientations to uniquely align $P$ (up to symmetries) from an arbitrary initial orientation (Figure 3d). Let us summarize the results:

Theorem 1 (Goldberg [7]) Let $P$ be a polygon whose convex hull has $n$ vertices. There is a sensorless control strategy $\mathcal{S}$ for a parallel-jaw gripper that aligns $P$ up to symmetries in $O(n)$ squeeze grasps. $\mathcal{S}$ can be computed in $O\left(n^{2}\right)$ time. ${ }^{2}$

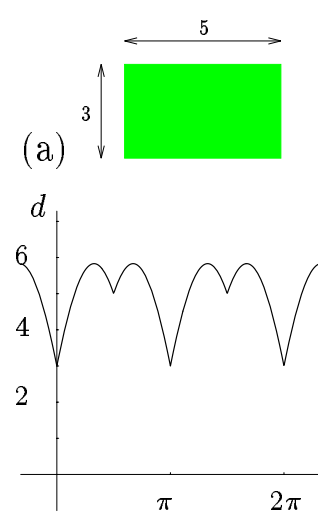

(b)

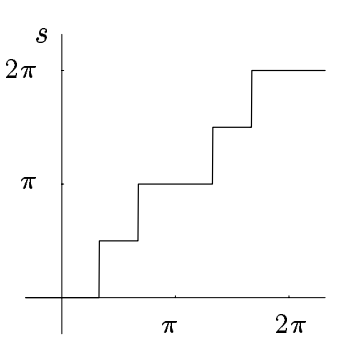

(c)
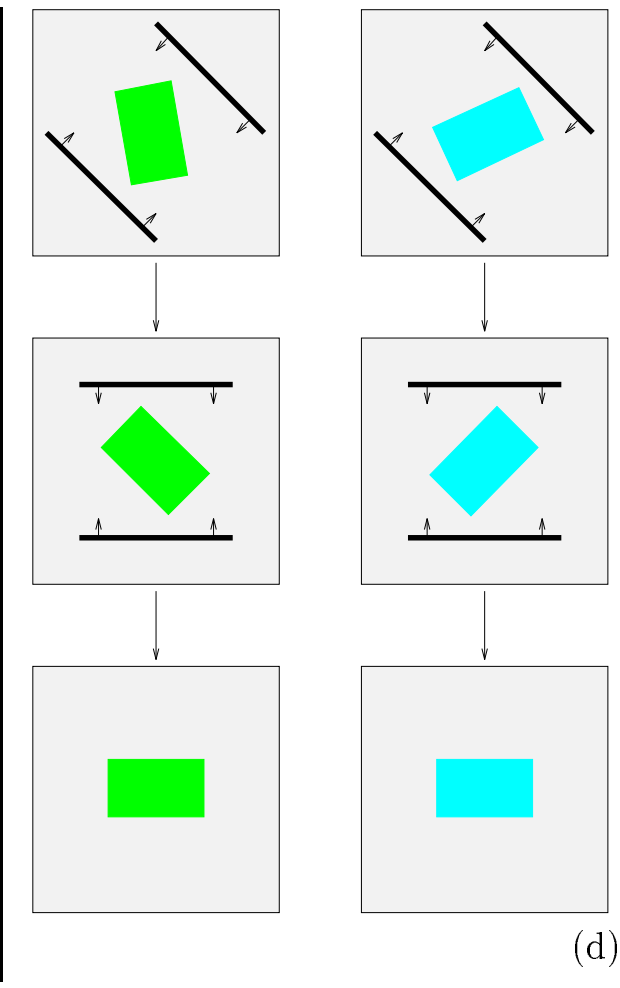

(d)
Figure 3: (a) Sample rectangle. (b) Diameter function. (c) Squeeze function. (d) Sensorless alignment strategy.

Now we show how to reduce a manipulation task with a microactuator array to an equivalent task with a parallel-jaw gripper. We make the following assumptions:

Simplicity: The moving part $P$ can be treated as a simple flat polygon.

Bilateral Symmetry: We have the following elementary actuator control scheme available: The array can be divided by a straight line $l$ such that all motion pixels on either side of $l$ push normally towards $l$.

Density: The generated forces can be described by a twodimensional vector field. This means that the individual microactuators are dense compared to the size of the moving part. (We will discuss later how to relax this assumption.)

We can now give a formal definition for an alignment strategy:

\footnotetext{
${ }^{2} O(k)$ ("order of $k$ ") denotes an upper bound on the complexity of a task with respect to an input parameter $k . O(n)$ means at most linear in $n, O\left(n^{2}\right)$ means at most quadratic in $n$.
} 
Definition 2 An alignment strategy $\mathcal{S}$ for an actuator array is a sequence of straight lines $\left(l_{1}, \ldots, l_{k}\right)$ such that assumption Bilateral Symmetry holds for all $l_{i}, 1 \leq i \leq k$.

Note that the system requires a clock that signals when enough time has elapsed for the object to reach its rest position.

An object will move towards the line $l$ and come to rest there (see also Furuhata et al. [6]). We are interested in the motion of an arbitrarily shaped part $P$. Let us call $P_{1}, P_{2}$ the regions of $P$ that lie to the left and to the right of $l$, respectively, and $C_{1}, C_{2}$ their centers of gravity. In a rest position both translational and rotational forces must be in equilibrium. We get the following two conditions:

I : The areas $P_{1}$ and $P_{2}$ must be equal.

II : The vector $C_{2}-C_{1}$ must be normal to $l$.

Definition $3 A$ median of a simple polygon $P$ is a straight line that divides $P$ into two parts of equal size.

Condition $\mathbf{I}$ says that $l$ is a median of $P . P$ has a motion component normal to $l$ if $\mathbf{I}$ does not hold. $P$ has a rotational motion component if II does not hold. $P$ is in equilibrium (stable or metastable) if and only if $\mathbf{I}$ and $\mathbf{I I}$ hold. See Figure 4 for an illustration.

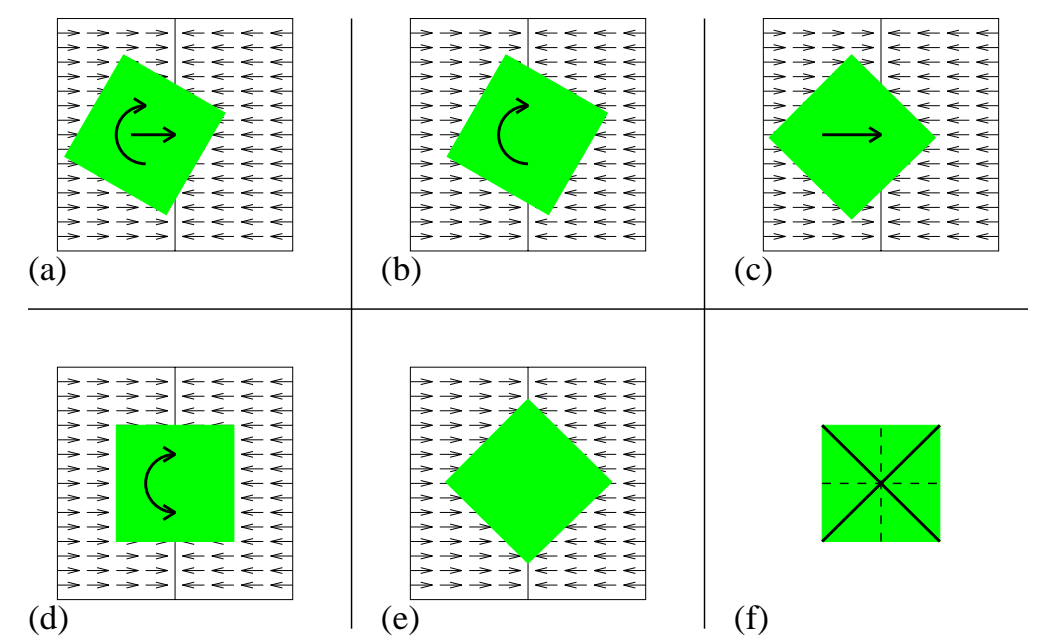

Figure 4: Square object on actuator array: (a) I not satisfied, II not satisfied. (b) I, not II. (c) not I, II. (d) I, II (metastable). (e) I, II (stable). (f) Stable (thick) and metastable (thin) medians of square.

For simplicity of presentation we make another assumption. This assumption will not hold in general, however it is not essential to the reduction and can be relaxed as described later. It corresponds exactly to the assumption that the parallel-jaw gripper performs pure squeeze grasps in which both jaws make contact with the part simultaneously [7].

2Phase: The motion of $P$ has two phases: (1) Pure translation towards $l$ until condition $\mathbf{I}$ is satisfied. (2) Motion until condition $\mathbf{I I}$ is satisfied without violating condition $\mathbf{I}$.

The following definition is in analogy with the diameter function above:

Definition 4 Let $\phi$ be the orientation of a simple polygon $P$ on an actuator array, and let us assume that condition $\mathbf{I}$ holds. The turn function $t: \phi \rightarrow\{-1,0,1\}$ describes the instantaneous rotational motion of $P . t(\phi)=1$ if $P$ will turn counterclockwise, $t(\phi)=-1$ if $P$ will turn clockwise, and $t(\phi)=0$ if $P$ is in equilibrium.

This definition immediately implies the following lemma:
Lemma 5 Let $P$ be a polygon with orientation $\phi$ on an actuator array such that conditions $\mathbf{I}$ and II hold. $P$ is stable if $t(\phi)=0, t(\phi+) \leq 0$, and $t(\phi-) \geq 0$. Otherwise $P$ is metastable.

Using this lemma we can identify all stable orientations, which allows us to construct the squeeze function of $P$ in analogy to Goldberg [7]:

Lemma 6 Let $P$ be a simple polygonal part on an actuator array $\mathcal{A}$ such that assumptions Simplicity, Bilateral SymMetry, Density, and 2Phase hold. Given the turn function $t$ of $P$, its corresponding squeeze function $s: \mathbb{S}^{1} \rightarrow \mathbb{S}^{1}$ is constructed as follows:

1. All stable orientations $\phi$ map to $\phi$.

2. All metastable orientations map (by convention) to the nearest right stable orientation.

3. All orientations $\phi$ with $t(\phi)=1(-1)$ map to the nearest right (left) stable orientation.

Then $s$ describes the transition of $P$ induced by $\mathcal{A}$.

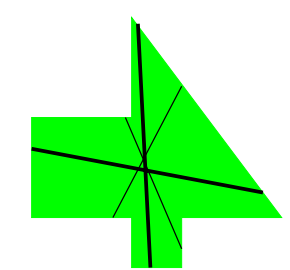

(a)

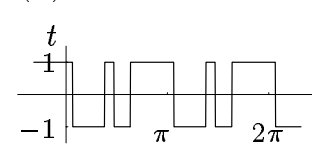

(b)

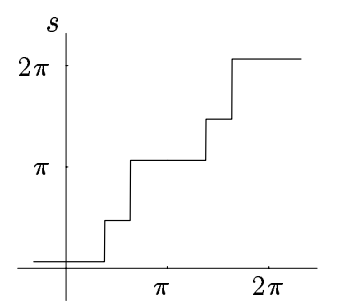

(c)

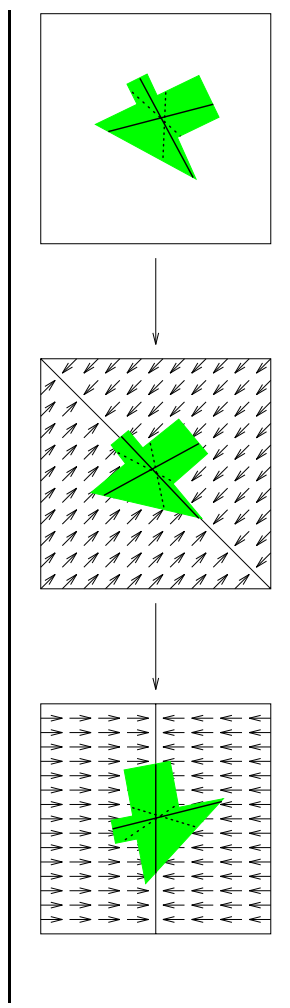

Figure 5: (a) Polygonal part; stable (thick line) and metastable (thin line) medians are also shown. (b) Turn function. (c) Squeeze function. (d) Alignment strategy for two arbitrary initial configurations.

See Figures $5 \mathrm{a}, \mathrm{b}, \mathrm{c}$ for an example. We can now complete the reduction from an actuator array to a parallel-jaw gripper:

Theorem 7 For a simple polygonal part $P$ and an actuator array $\mathcal{A}$ there exists an alignment strategy $\mathcal{S}=\left(l_{1}, \ldots, l_{k}\right)$ that uniquely aligns $P$ up to symmetries.

Proof: We can get a formula for the turn function $t$ of $P$ by taking the sign of the dot product between the direction of the line $l$ and the line connecting $C_{1}$ and $C_{2}$. Straightforward algebra shows that this product can be written as a piecewise rational function of fixed low degree, with $O\left(n^{2}\right)$ pieces for general simple polygons, and $O(n)$ pieces for convex polygons. From $t$ we can construct the squeeze function $s$ (Lemma 6 ) within the same time bounds. Then the alignment strategy $\mathcal{S}$ is obtained by using Goldberg's algorithm [7] (Theorem 1). $\square$

From the proof we can obtain complexity bounds for microactuator alignment strategies [3]: If $P$ is a $n$-gon, the algorithm 
runs in time $O\left(n^{4}\right)$ and produces a strategy $\mathcal{S}=\left(l_{1}, \ldots, l_{k}\right)$ of length $k=O\left(n^{2}\right)$. If $P$ is convex the running time is $O\left(n^{2}\right)$ and $k=O(n)$.

Finally let us reconsider two of the assumptions made earlier in this section. Relaxing 2PнASE corresponds to allowing pushsqueeze grasps for the parallel-jaw gripper [7] in which one jaw pushes $P$ before the second jaw makes contact with $P$. The squeeze function must be replaced by a shift-squeeze function which takes combined translational and rotational motions into account. However neither the (meta-)stable orientations of $P$ nor the complexity of the turn function will change, so the complexity of the generated strategy remains the same. Similar constructions seem possible to find reductions to conveyer belts [14] or tilting trays [5].

If we want to relax assumption Density we need to model the mechanics of individual actuators and understand their interaction. Relaxing Density is necessary to manipulate parts that are only slightly larger than the actuators. This is discussed in the following section.

\section{MICROSCOPIC MODEL FOR ACTUATOR CONTACT}

In this section we develop a model for the mechanics of microactuators. We make use of limit surfaces [8] that describe anisotropic frictional contact. We extend the model to active contacts and describe fast algorithms to compute the combined effect of many actuators.

Limit Surfaces. Assume we have a part $P$ that moves on top of the actuator array. The limit surface $\mathcal{L}$ in load space (forces $F_{x}$ and $F_{y}$, moment $M$ ) fully describes the relationship between generalized velocity $v$, effective applied load $F_{\text {eff }}$, and frictional load $F_{r}$ of the moving part. It is based on the Maximum Work Inequality which is an engineering assumption commonly used when modeling friction or plasticity $[8,13]$. The Maximum Work Inequality generalizes Coulomb's friction law to anisotropic rate-independent friction. The following properties of limit surfaces are useful [8]:

1. $\mathcal{L}$ is a closed convex surface in load space.

2. $\mathcal{L}$ contains all possible frictional loads $F_{r}$ on $P$.

3. If $v \neq 0, F_{r} \in \mathcal{L}$ and $-v$ is normal to $\mathcal{L}$ at $F_{r}$.

4. $F_{r}$ is the vector inside or on $\mathcal{L}$ such that the length of $F_{r}-F_{e f f}$ is minimal.

5. In the special case when the moment component $M$ of $F_{r}$ is $0, \mathcal{L}$ is a limit curve in $\left(F_{x}-F_{y}\right)$ load space.

See Figure 6 for examples. It follows that the inside of $\mathcal{L}$ contains all loads $F_{\text {eff }}$ that can be applied to $P$ without setting it in motion, and if $F_{\text {eff }} \neq 0$ and $v \neq 0, F_{r}$ is determined uniquely. But note that for given $v, F_{r}$ is not unique if $\mathcal{L}$ has
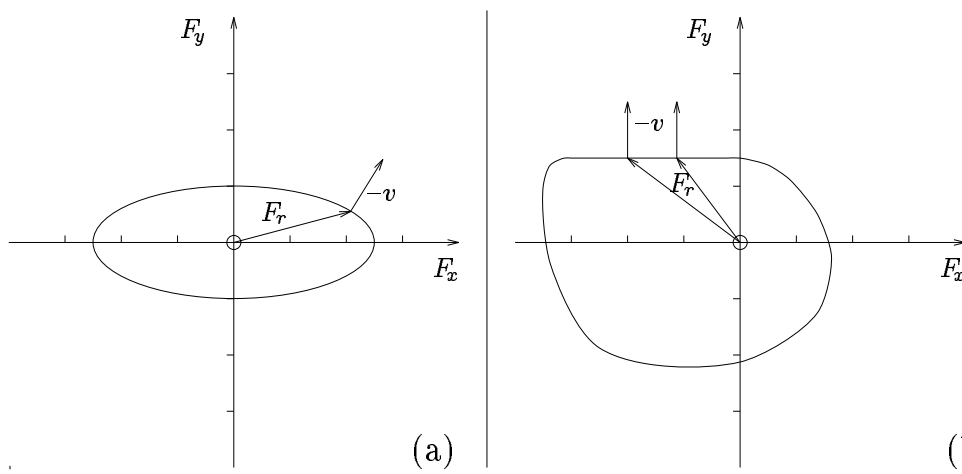

Figure 6: Limit curves: (a) $-v$ is always normal to $\mathcal{L}$ at $F_{r}$. (b) $F_{r}$ can be nonunique for a specific given $v$.

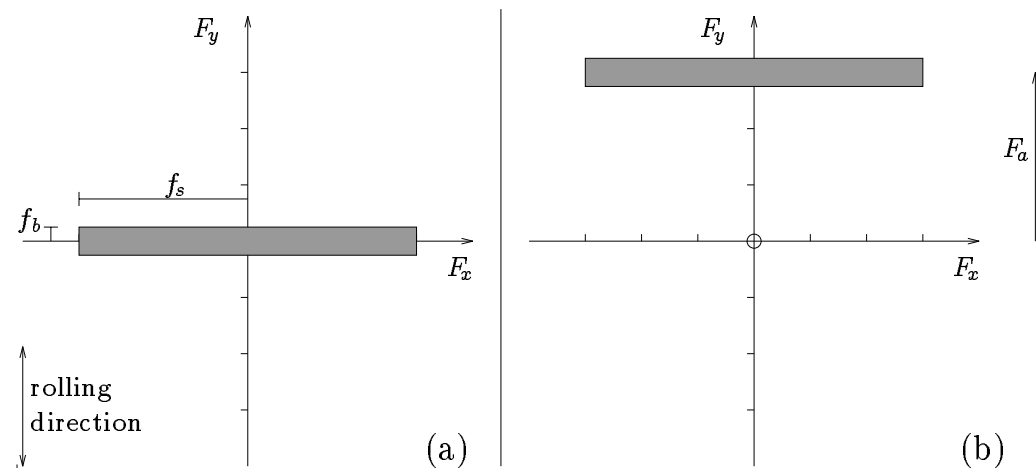

Figure 7: Limit curve of (a) passive wheel, (b) driven wheel.

a flat face with normal $-v$. Similarly for given $F_{r}, v$ is not unique if $\mathcal{L}$ has a vertex or edge at $F_{r}$. This indeterminacy can be resolved by taking the inertia of $P$ into account [8].

Consider as an example Figure 7a. The anisotropic behavior of a wheel can be modeled with a long rectangular limit curve which gives low bearing friction $f_{b}$ in the rolling direction and high sideways friction $f_{s}$.

Active Contacts. We now extend the limit surface model to "active" contacts that apply loads to $P$.

Definition 8 The active limit surface $\mathcal{L}$ in load space is the set of loads that can be applied to $P$ without resulting in motion of $P$.

This definition includes limit surfaces for passive contacts, but it allows us to model for example a wheel driven by some torque $\tau_{a}$. Figure $7 \mathrm{~b}$ shows that if no additional load is applied the wheel will move in $y$ direction, accelerated by $F_{a}$ minus the bearing friction $f_{b}$ (where $F_{a}$ is such that $\tau_{a}=r \times F_{a}$, and $r$ is the radius of the wheel). In general we get motion if the origin of load space $\mathcal{O}$ lies outside of $\mathcal{L}$.

For the wheel accelerated with torque $\tau_{a}=r \times F_{a}$ the limit curve simply shifts in load space by $F_{a}$. For our actuators we expect the shape of active and passive limit surface to be different because of interactions between friction and oscillation. However, because the limit surface will represent the time average over frictional contacts, we believe that the theory of limit surfaces is a valid model.

Combining Limit Surfaces. We have already noted one advantage of limit surfaces: they offer a uniform, purely geometric representation of contact properties. In addition, multiple contacts can be described by a single joint limit surface. Goyal and Ruina [8] have shown that this joint limit surface is the convolution (Minkowski sum) of the individual limit surfaces. There exist fast algorithms from computational geometry [9] to compute this convolution. They are described in more detail in our technical report [3]. Thus limit surfaces offer a technique to efficiently determine and represent the behavior of an entire actuator array.

For illustration, Figure 8 shows a rigid bar with two point contacts and Coulomb friction, and their corresponding limit surfaces. The individual surfaces are flat because they can generate no moment about the contact point, but tilted because they can generate moments about the center of mass. The tilt angle $\theta$ can be determined by $\tan \theta=\frac{|M|}{F \mid}=\frac{|r \times F|}{|F|}=|r|$, so for $|r|=1$ we get $\theta=45^{\circ}$. Figure 9 shows the combined limit surface.

Motion Prediction. For simplicity let us first consider the "upside-down" case where the actuator array "walks" on a homogeneous flat surface. The contacts and thus the limit surface 


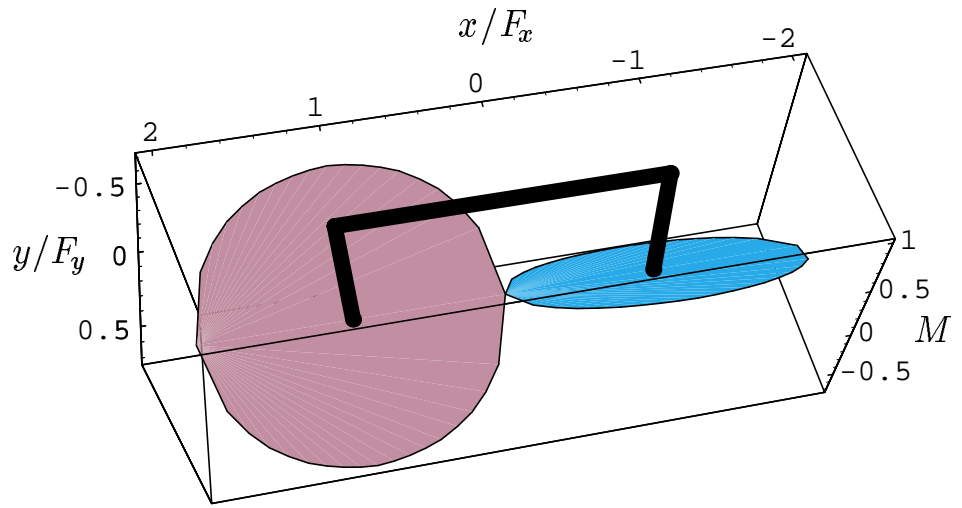

Figure 8: Bar with two point contacts at $(1,0)$ and $(-1,0)$, and their corresponding limit surfaces with respect to the center of mass $(0,0)$.

$\mathcal{L}$ are fixed with respect to a coordinate system attached to the actuator array. If the origin of load space $\mathcal{O}$ is inside $\mathcal{L}$ there will be no motion. Otherwise the generated force $F$ is the point on $\mathcal{L}$ closest to $\mathcal{O}$. At that point the surface normal is parallel to $F$, so velocity and accelerating force are parallel. The walker will move on a straight line or a circle. This is not unexpected for a fixed actuator strategy on a homogeneous surface.

Now consider the case where the object is on top of the actuator array. There are two major differences to the previous case: When the object moves (1) some actuators loose contact, others make contact, (2) the induced moment changes. (1) requires periodic updates of the limit surface. (2) results in stretching of the limit surface along the moment axis. Our corresponding technical report [3] gives details on how to handle these cases efficiently. There, we outline a simple yet efficient motion prediction algorithm: Numerically integrate the velocities computed as described above, and update the limit surface $\mathcal{L}$ accordingly. Each integration step can be done in constant time. Each update of $\mathcal{L}$ is linear in the complexity of $\mathcal{L}$.

Motion Planning. The shape of the limit surface is determined by the activation pattern of the actuator array, which depends on each individual actuator. The limit surface gives us a geometric representation of the forces and velocities generated with a specific actuator activation pattern. Though theoretically possible, there are practical limitations on using limit surfaces to plan microscopic manipulation strategies due to the combinatorial complexity and mechanical uncertainty. However, the microscopic model will prove important to analyze

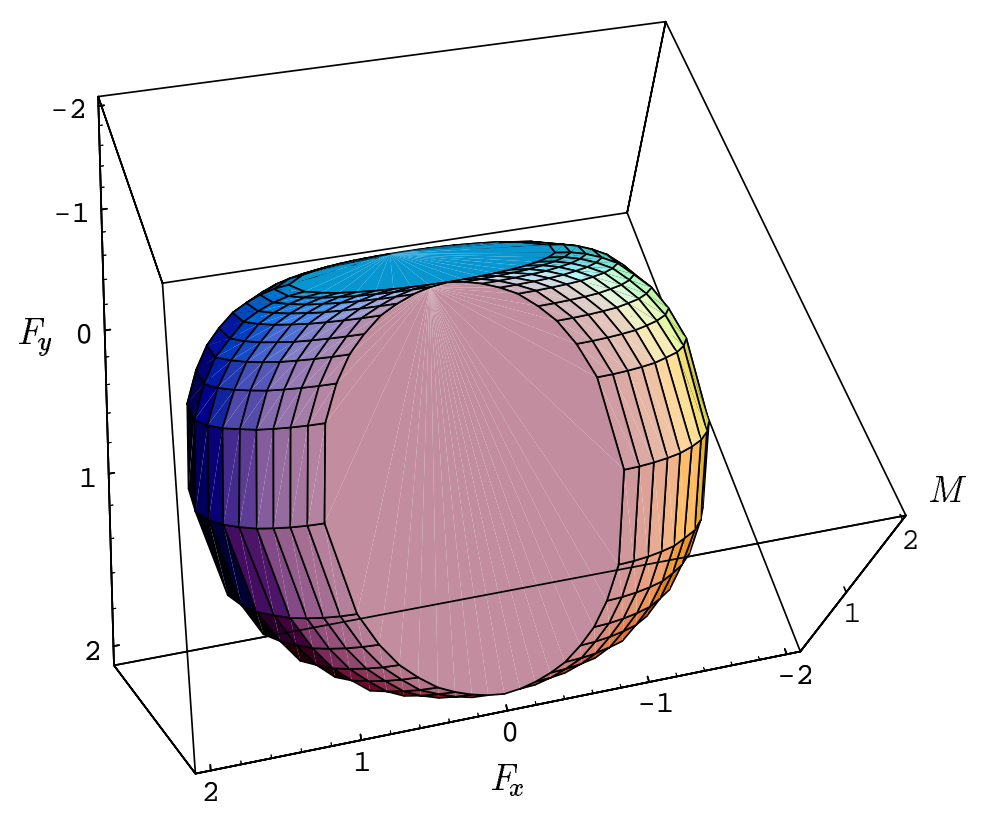

Figure 9: Combined limit surface for the object in Figure 8. and verify strategies before fabrication. It forms the link between "actuator macros" (Section 3) that predict the global behavior of manipulation strategies, and individual microfabricated mechanisms (Section 5).

\section{FABRICATION OF ACTUATOR ARRAY}

Design Aspects. Figure 10 shows a schematic cross section of an actuator with poles on one edge. $h$ is the clearance between resonator and trench, $x_{1}$ the distance between pole and rotational axis, $x_{2}$ the width of the other side of the resonator, $z$ the height of the poles, and $\phi$ the deflection of the resonator. If the actuator is inactive, the moving object will rest on the poles. In case it is active and the poles are moving upward we assume that the poles push the object up and sideways. During the downward motion we assume that the object keeps its horizontal position (e.g. because other actuators are holding it) but moves straight down (due to gravity). This results in a stepwise sideways motion with each oscillation of the actuator. We want to optimize this motion.

First note that we should choose $x_{1} \approx x_{2}=\bar{x} . x_{1} \gg x_{2}$ would decrease the force and horizontal motion at the pole tips. $x_{1}<x_{2}$ yields a force-motion tradeoff (lever). $x_{1} \ll x_{2}$ is unfavorable because then the maximum deflection of the pole tip would be smaller than the deflection of the other edge of the resonator.

Let $\Delta x$ be the horizontal component of the motion of a pole tip when it moves from $\phi=0$ to maximum deflection $\widehat{\phi}$. Then $\Delta x=z \sin \widehat{\phi}+x_{1}(1-\cos \widehat{\phi})$.

The motion generated by the device is $\frac{\Delta x}{\Delta t}=\Delta x \cdot f$, where $f$ is its resonance frequency, and both $\Delta x$ and $f$ are functions of $\bar{x}$. It can be shown that the derivative of $\Delta x \cdot f$ is always non-positive. This means that decreasing $\bar{x}$ improves the performance of the actuator.

In summary, the horizontal motion is optimized by choosing $h$ and $z$ as large as permitted by the fabrication process. Then choose $x_{1} \approx x_{2}$ as small as possible. Obviously in practice still $x_{2} \gg h$, otherwise $\hat{\phi}$ will not be reached. Note that this result means that we have to maximize the aspect ratio of our design. This has an additional advantage: If the horizontal size is reduced we can pack more devices per area. This yields further performance improvement.

Fabrication Process. The actuators are etched out of a singlecrystal silicon wafer in a SCREAM (Single Crystal Reactive Etching and Metallization) process $[18,19,12]$. A dry etch releases grid structures with beams of up to $1 \mu \mathrm{m}$ width. Metal electrodes are deposited during a self-aligning aluminum evap-

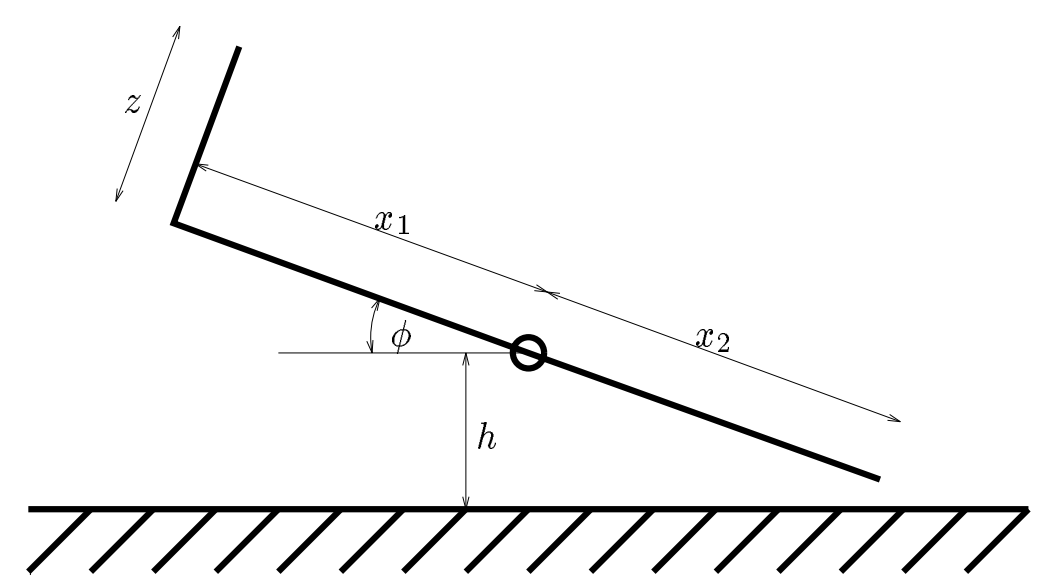

Figure 10: Schematic cross section of actuator (not to scale).

Proc. Seventh IEEE International Workshop on Micro Electro Mechanical Systems, Oiso, Japan, January 1994. 
oration. Single beams are up to $5 \mu \mathrm{m}$ high, with $\approx 3 \mu \mathrm{m}$ clearance underneath. This clearance allows the out-of-plane motion of the resonator grid. For cleaning and improvement of levitation it is conceivable to combine the resonator with air nozzles as described in Section 2 [15]. The fabrication can be done in one to two weeks in the National Nanofabrication Facility (NNF) at Cornell University.

Efficiency. We have analyzed a resonator of size $50 \times 50 \mu \mathrm{m}^{2}$ with clearance $h=3 \mu \mathrm{m}$ using the finite element simulator Coulom b [10]. Similar actuators are shown in Figures 1 and 2. The vertical force generated is $2.8 \cdot 10^{-7} \mathrm{~N}$ when a voltage of $50 \mathrm{~V}$ is applied. Assuming that the entire device uses a total area of $100 \times 100 \mu \mathrm{m}^{2}$ we get $2.8 \cdot 10^{-11} \frac{\mathrm{N}}{\mu \mathrm{m}^{2}}$. This is almost two orders of magnitude higher than the specific weight of paper $80 \frac{g}{m^{2}} \cong 8 \cdot 10^{-13} \frac{N}{\mu m^{2}}$. This indicates that our devices are strong enough to do practical manipulation tasks. Downscaling of the devices will further improve this ratio, because the force decreases linearly with the scaling, while the actuator density grows quadratically with decreasing scale.

Results. A wide variety of resonators has been built and tested in the NNF at Cornell University (see Figure 2), yielding information on the optimal design of actuators and material properties such as stiffness, structural sturdiness, and internal stresses. In experiments with single actuators we observed motion of powder particles. A prototype of actuator arrays is currently being built.

\section{CONCLUSIONS AND FUTURE WORK}

We have outlined a theory of manipulation and control for microfabricated actuator arrays that applies concepts from robotics to the field of MEMS. We believe that joint efforts in these fields are important for future MEMS of high complexity, and will prove fruitful for both areas.

The next steps of laboratory work will include the fabrication of a prototype array with a large number of microactuators, the experimental characterization of the limit surface of a microactuator, and experiments on micromanipulation to evaluate and validate our model.

The ideas presented here extend work in our group on parallel, distributed robotics [4] to massively parallel systems with similar, relatively simple individual components (DMMS [17]). Future work will include exploration of the limitations of macroscopic manipulation strategies due to the quantized forces generated by motion pixels, and the determination of quantitative error estimates. Other goals are the development and analysis of additional macroscopic strategies ("actuator macros"), and a more detailed design and implementation of motion prediction algorithms. The low-temperature SCREAM process is compatible with conventional VLSI fabrication, which allows mechanisms and logic on one chip. This combination would make complex control strategies possible. Finally we also hope to address the case where the actuator array "walks" on a flat surface. This could conceivably lead to walking or selfassembling chips.

\section{REFERENCES}

[1] Analog Devices, Inc., Norwood, MA 02062. Introducing the ADXL50 Micromachined Accelerometer Sensor, 1991.

[2] M. Ataka, A. Omodaka, and H. Fujita. A biomimetic micro motion system. In Transducers - Digest Int. Conf. on Solid-
State Sensors and Actuators, pages 38-41, Pacifico, Yokohama, Japan, June 1993.

[3] K.-F. Böhringer, B. R. Donald, R. Mihailovich, and N. C. MacDonald. A geometric theory of manipulation and control for microfabricated actuator arrays. Technical Report 93-87, Cornell University, Mathematical Sciences Institute, Ithaca, NY 14853, Nov. 1993.

[4] B. R. Donald, J. Jennings, and D. Rus. Towards a theory of information invariants for cooperating autonomous mobile robots. In International Symposium of Robotics Research, Hidden Valley, PA, Oct. 1993.

[5] M. A. Erdmann and M. T. Mason. An exploration of sensorless manipulation. IEEE Journal of Robotics and Automation, 4(4), Aug. 1988.

[6] T. Furuhata, T. Hirano, and H. Fujita. Array-driven ultrasonic microactuators. In Transducers - Digest Int. Conf. on Solid-State Sensors and Actuators, pages 1056-1059, Montreux, France, June 1991.

[7] K. Y. Goldberg. Orienting polygonal parts without sensing. Algorithmica, 10(2/3/4):201-225, August/September/October 1993.

[8] S. Goyal and A. Ruina. Relation between load and motion for a rigid body sliding on a planar surface with dry friction: Limit surfaces, incipient and asymptotic motion. In WEAR, 1988.

[9] L. J. Guibas and R. Seidel. Computing convolutions by reciprocal search. In Proceedings of the ACM Symposium on Computational Geometry, Urbana, IL, 1988.

[10] Integrated Engineering Software Inc. Coulomb, 1989. Users and Technical Manual, Version 2.1.

[11] S. Konishi and H. Fujita. A conveyance system using air flow based on the concept of distributed micro motion systems. In Transducers - Digest Int. Conf. on Solid-State Sensors and Actuators, pages 28-31, Pacifico, Yokohama, Japan, June 1993.

[12] R. E. Mihailovich, Z. L. Zhang, K. A. Shaw, and N. C. MacDonald. Single-crystal silicon torsional resonators. In Proc. IEEE Workshop on Micro Electro Mechanical Systems, pages 155-160, Fort Lauderdale, FL, Feb. 1993.

[13] M. A. Peshkin and A. C. Sanderson. Minimization of energy in quasistatic manipulation. In Proc. IEEE Int. Conf. on Robotics and Automation, pages 421-426, Philadelphia, PA, 1988.

[14] M. A. Peshkin and A. C. Sanderson. Planning robotic manipulation strategies for workpieces that slide. IEEE Journal of Robotics and Automation, 4(5):524-531, Oct. 1988.

[15] K. S. J. Pister, R. Fearing, and R. Howe. A planar air levitated electrostatic actuator system. In Proc. IEEE Workshop on Micro Electro Mechanical Systems, pages 67-71, Napa Valley, California, Feb. 1990.

[16] J. B. Sampsell. The digital micromirror device and its application to projection displays. In Transducers - Digest Int. Conf. on Solid-State Sensors and Actuators, pages 24-27, Pacifico, Yokohama, Japan, June 1993.

[17] N. Takeshima and H. Fujita. Design and control of systems with microactuator arrays. In Proc. IEEE Workshop in Advanced Motion Control, pages 219-232, Yokohama, Japan, Mar. 1990.

[18] Z. L. Zhang and N. C. MacDonald. An RIE process for submicron, silicon electromechanical structures. Journal of Micromechanics and Microengineering, 2(1):31-38, Mar. 1992.

[19] Z. L. Zhang and N. C. MacDonald. Fabrication of submicron high-aspect-ratio GaAs actuators. Journal of Microelectromechanical Systems, 2(2):66-73, June 1993.

HISTORICAL NOTES AND ACKNOWLEDGEMENTS. The authors would like to note that Goyal and Ruina were aware of algorithms for the construction of limit surfaces but did not mention them in their article [8].

We would like to thank John Canny, Ken Goldberg, and Steve Vavasis for useful discussions and comments, and staff and students in the National Nanofabrication Facility at Cornell University for their assistance. 